\title{
RESEARCH
}

\section{A study of elliptic gamma function and allies}

Vicenţiu Paşol ${ }^{1}$ and Wadim Zudilin $2,3,4^{*}$ (D)

*Correspondence:
w.zudilin@math.ru.nl;
wadim.zudilin@newcastle.edu.au;
wzudilin@gmail.com
2Department of Mathematics,
IMAPP, Radboud University, P.O.
Box9010,6500 GL Nijmegen,
The Netherlands
Full list of author information is
available at the end of the article
Dedicated to Don Zagier, in
admiration of his insights on
modular, elliptic and
polylogarithmic functions.

${ }^{*}$ Correspondence: w.zudilin@math.ru.n: wzudilin@gmail.com

2Department of Mathematics, IMAPP, Radboud University, P.O.

Box 9010, 6500 GL Nijmegen

available at the end of the article

ed to Don Zagier, in

polylogarithmic functions.

\section{Abstract}

We study analytic and arithmetic properties of the elliptic gamma function

$$
\prod_{m, n=0}^{\infty} \frac{1-x^{-1} q^{m+1} p^{n+1}}{1-x q^{m} p^{n}}, \quad|q|,|p|<1,
$$

in the regime $p=q$, in particular, its connection with the elliptic dilogarithm and a formula of $\mathrm{S}$. Bloch. We further extend the results to more general products by linking them to non-holomorphic Eisenstein series and, via some formulae of D. Zagier, to elliptic polylogarithms.

Keywords: Theta function, Elliptic gamma function, Elliptic dilogarithm, Elliptic polylogarithm

\section{Introduction}

For complex $z$ and $\tau$ with $\operatorname{Im} \tau>0$, set $x=e^{2 \pi i z}$ and $q=e^{2 \pi i \tau}$. Transformation properties of the so-called short theta function

$$
\theta_{0}(z ; \tau):=\prod_{m=0}^{\infty}\left(1-x^{-1} q^{m+1}\right)\left(1-x q^{m}\right)
$$

under the action of the modular group are well understood. In view of its transparent invariance under translation $\tau \mapsto \tau+1$, the main source of the modular action originates from the $\tau$-involution

$$
z \mapsto \hat{z}=\frac{z}{\tau}, \quad \tau \mapsto \hat{\tau}=-\frac{1}{\tau} .
$$

The related classical transformation of $\theta_{0}(z ; \tau)$ can be recorded as

$$
q^{1 / 12} x^{-1 / 2} \theta_{0}(z ; \tau)=i e^{-\pi i z \hat{z}} \hat{q}^{1 / 12} \hat{x}^{-1 / 2} \theta_{0}(\hat{z} ; \hat{\tau})
$$

(see, for example, [3, Section 2]), where we define $\hat{x}=e^{2 \pi i \hat{z}}$ and $\hat{q}=e^{2 \pi i \hat{t}}$.

Less is known about modular properties of the related product

$$
\theta_{1}(z ; \tau):=\prod_{m=0}^{\infty} \frac{\left(1-x^{-1} q^{m+1}\right)^{m+1}}{\left(1-x q^{m}\right)^{m}},
$$

(c) The Author(s) 2018. This article is distributed under the terms of the Creative Commons Attribution 4.0 International License (http://creativecommons.org/licenses/by/4.0/), which permits unrestricted use, distribution, and reproduction in any medium, provided you give appropriate credit to the original author(s) and the source, provide a link to the Creative Commons license, and indicate if changes were made. 
which naturally comes as the $\sigma=\tau$ specialisation of the elliptic gamma function

$$
\Gamma(z ; \tau, \sigma):=\prod_{m, n=0}^{\infty} \frac{1-x^{-1} q^{m+1} p^{n+1}}{1-x q^{m} p^{n}}, \quad \text { where } p=e^{2 \pi i \sigma},
$$

introduced by Ruijsenaars [5] (see also [3,4]). Namely, we have

$$
\theta_{1}(z ; \tau)=\theta_{0}(z ; \tau) \Gamma(z ; \tau, \tau)=\Gamma(z+\tau ; \tau, \tau) .
$$

A known functional equation of the elliptic gamma function [3, Theorem 4.1] represents an $\mathrm{SL}_{3}(\mathbb{Z})$ symmetry of $\Gamma(z ; \tau, \sigma)$. The problem of determining its behaviour in the regime $\sigma=\tau$ under $\mathrm{SL}_{2}(\mathbb{Z})$ transformations is specifically addressed in [2], where the (logarithm of the) infinite product is related to the elliptic dilogarithm via a formula of S. Bloch [1].

Our principal aim in this note is recasting analytic and arithmetic (modular) properties of the function $\theta_{1}(z ; \tau)$ and its relatives, in particular, linking them to non-holomorphic Eisenstein series and the elliptic dilogarithm. This programme is carried out in Sects. 2-4; it gives a new proof of Bloch's formula and related results from [2]. In Sect. 5 we go further to discuss similar features of products that generalise ones for $\theta_{0}$ and $\theta_{1}$; their relationship with non-holomorphic Eisenstein series and formulae from [7] allow us to link them to elliptic polylogarithms.

For future record, notice that iterating the transformation $(z, \tau) \mapsto(\hat{z}, \hat{\tau})$ twice maps $(z, \tau)$ to $(-z, \tau)$ and that

$$
\theta_{1}(-z ; \tau)=\frac{1}{\theta_{1}(z ; \tau)} \text { and } \theta_{0}(-z ; \tau)=-x^{-1} \theta_{0}(z ; \tau)
$$

\section{Period functions}

A natural way of measuring failure of weight $k$ modular behaviour under the transformation $(z, \tau) \mapsto(\hat{z}, \hat{\tau})$ for a function $f(z, \tau)$ is through the period function

$$
g(z, \tau)=g_{k}(z, \tau):=f(\hat{z}, \hat{\tau})-\tau^{k} f(z, \tau) .
$$

\section{Lemma 1 We have}

$$
\tau^{k} g(\hat{z}, \hat{\tau})+(-1)^{k} g(z, \tau)=\tau^{k}\left(f(-z, \tau)-(-1)^{k} f(z, \tau)\right) .
$$

Observe that the expression in the parentheses on the right-hand side measures the failure of $k$-parity of $f(z, \tau)$.

Proof We only use $(\hat{\hat{z}}, \hat{\hat{\tau}})=(-z, \tau)$ and $\tau \hat{\tau}=-1$ :

$$
\begin{aligned}
\tau^{k} g(\hat{z}, \hat{\tau})-g(z, \tau) & =\tau^{k}\left(f(-z, \tau)-\hat{\tau}^{k} f(\hat{z}, \hat{\tau})\right)+(-1)^{k}\left(f(\hat{z}, \hat{\tau})-\tau^{k} f(z, \tau)\right) \\
& =\tau^{k}\left(f(-z, \tau)-(-1)^{k} f(z, \tau)\right) .
\end{aligned}
$$

The lemma and the parity relation for $\ln \theta_{1}(z ; \tau)$ in (3) imply the following.

Lemma 2 The function

$$
T(z ; \tau)=\tau \ln \theta_{1}(z ; \tau)-\ln \theta_{1}(\hat{z} ; \hat{\tau})
$$

satisfies the functional equation

$$
T(\hat{z} ; \hat{\tau})=\tau^{-1} T(z ; \tau) .
$$


Furthermore, we can relate the function $T(z ; \tau)$ to the dilogarithm function

$$
\mathrm{Li}_{2}(x)=-\int_{0}^{x} \ln (1-t) \frac{\mathrm{d} t}{t} .
$$

Lemma 3 The function (4) admits the following representation:

$$
\begin{aligned}
T(z ; \tau)= & \frac{\pi i(\tau-2 z)\left(1+2 \tau z-2 z^{2}\right)}{12 \tau}+z \ln \theta_{0}(z ; \tau) \\
& -\frac{1}{2 \pi i} \sum_{m=0}^{\infty}\left(\operatorname{Li}_{2}\left(x^{-1} q^{m+1}\right)-\operatorname{Li}_{2}\left(x q^{m}\right)\right) .
\end{aligned}
$$

Proof As shown in the proof of Theorem 5.2 in [3],

$$
\begin{aligned}
\ln \theta_{1}(z ; \tau)= & \ln \theta_{0}(z ; \tau)+\ln \Gamma(z ; \tau, \tau) \\
= & -\pi i \lambda(z ; \tau)+\ln \frac{\theta_{0}(z ; \tau)}{\theta_{0}(\hat{z} ; \hat{\tau})} \\
& +(\hat{\tau}-\hat{z}) \sum_{k=1}^{\infty} \frac{\left(\hat{x}^{-1} \hat{q}\right)^{k}}{k\left(1-\hat{q}^{k}\right)}-\hat{z} \sum_{k=1}^{\infty} \frac{\hat{x}^{k}}{k\left(1-\hat{q}^{k}\right)} \\
& +\frac{1}{2 \pi i} \sum_{k=1}^{\infty} \frac{\hat{x}^{k}-\left(\hat{x}^{-1} \hat{q}\right)^{k}}{k^{2}\left(1-\hat{q}^{k}\right)}-\hat{\tau} \sum_{k=1}^{\infty} \frac{\hat{q}^{k}\left(\hat{x}^{k}-\left(\hat{x}^{-1} \hat{q}\right)^{k}\right)}{k\left(1-\hat{q}^{k}\right)^{2}}
\end{aligned}
$$

where

$$
\lambda(z ; \tau)=\frac{z^{3}}{3 \tau^{2}}-\frac{2 \tau-1}{2 \tau^{2}} z^{2}+\frac{(\tau-1)(5 \tau-1)}{6 \tau^{2}} z-\frac{(\tau-2)(2 \tau-1)}{12 \tau}
$$

and the assumptions $|\hat{x}|,\left|\hat{x}^{-1} \hat{q}\right|<1$ are made to ensure convergence. (The latter can be dropped in the final result by appealing to the analytic continuation in $z$.) Recalling the transformation (2), using

$$
\frac{1}{1-\hat{q}^{k}}=\sum_{m=0}^{\infty} \hat{q}^{m k} \quad \text { and } \quad \frac{\hat{q}^{k}}{\left(1-\hat{q}^{k}\right)^{2}}=\sum_{m=0}^{\infty} m \hat{q}^{m k},
$$

interchanging summation and summing over $k$, we obtain

$$
\begin{aligned}
\ln \theta_{1}(z ; \tau)= & -\pi i\left(\lambda(z ; \tau)-\frac{1}{2}+\frac{z^{2}}{\tau}+\frac{\tau}{6}-z+\frac{1}{6 \tau}+\frac{z}{\tau}\right) \\
& +\hat{z} \sum_{m=0}^{\infty}\left(\ln \left(1-\hat{x}^{-1} \hat{q}^{m+1}\right)+\ln \left(1-\hat{x} \hat{q}^{m}\right)\right) \\
& -\hat{\tau} \sum_{m=0}^{\infty}\left((m+1) \ln \left(1-\hat{x}^{-1} \hat{q}^{m+1}\right)-m \ln \left(1-\hat{x} \hat{q}^{m}\right)\right) \\
& -\frac{1}{2 \pi i} \sum_{m=0}^{\infty}\left(\operatorname{Li}_{2}\left(\hat{x}^{-1} \hat{q}^{m+1}\right)-\operatorname{Li}_{2}\left(\hat{x} \hat{q}^{m}\right)\right) \\
= & \frac{\pi i}{12}\left((1+2 z)-\frac{2 z(1+z)(1+2 z)}{\tau^{2}}\right)+\hat{z} \ln \theta_{0}(\hat{z} ; \hat{\tau})-\hat{\tau} \ln \theta_{1}(\hat{z} ; \hat{\tau}) \\
& -\frac{1}{2 \pi i} \sum_{m=0}^{\infty}\left(\operatorname{Li}_{2}\left(\hat{x}^{-1} \hat{q}^{m+1}\right)-\operatorname{Li}_{2}\left(\hat{x} \hat{q}{ }^{m}\right)\right) .
\end{aligned}
$$


(This formula can be alternatively derived from logarithmically differentiating identity

(2) with respect to $\tau$ and further integrating the result with respect to $z$.) Substituting $(z / \tau,-1 / \tau)$ for $(z, \tau)$ translates the result into

$$
\begin{aligned}
\tau \ln \theta_{1}(z ; \tau)-\ln \theta_{1}(\hat{z} ; \hat{\tau})= & \frac{\pi i(\tau-2 z)\left(1+2 \tau z-2 z^{2}\right)}{12 \tau}+z \ln \theta_{0}(z ; \tau) \\
& -\frac{1}{2 \pi i} \sum_{m=0}^{\infty}\left(\operatorname{Li}_{2}\left(x^{-1} q^{m+1}\right)-\operatorname{Li}_{2}\left(x q^{m}\right)\right),
\end{aligned}
$$

the desired relation.

\section{Non-holomorphic modularity}

Denote

$$
A=A(z, \tau):=\frac{z-\bar{z}}{\tau-\bar{\tau}} \in \mathbb{R},
$$

so that

$$
\hat{A}=A(\hat{z}, \hat{\tau}):=\frac{z \bar{\tau}-\bar{z} \tau}{\tau-\bar{\tau}} \in \mathbb{R}
$$

and $z=A \tau-\hat{A}$. Define

$$
Q(z ; \tau):=q^{B_{3}(A) / 3} \prod_{m=0}^{\infty} \frac{\left(1-x q^{m}\right)^{m+A}}{\left(1-x^{-1} q^{m+1}\right)^{m+1-A}}=\frac{q^{B_{3}(A) / 3} \theta_{0}(z ; \tau)^{A}}{\theta_{1}(z ; \tau)},
$$

where $B_{3}(t):=t^{3}-\frac{3}{2} t^{2}+\frac{1}{2} t$ is the third Bernoulli polynomial, $B_{3}(1-t)=-B_{3}(t)$, and

$$
F_{+}(z ; \tau):=\ln Q(\hat{z} ; \hat{\tau})-\tau \ln Q(z ; \tau), \quad F_{-}(z ; \tau):=\ln \overline{Q(\hat{z} ; \hat{\tau})}-\tau \ln \overline{Q(z ; \tau) .}
$$

It follows then from Lemma 1 and the parity relations (3) that

$$
\begin{aligned}
\tau F_{+}(\hat{z} ; \hat{\tau})-F_{+}(z ; \tau) & =\tau(\ln Q(-z ; \tau)+\ln Q(z ; \tau)) \\
& =\frac{2 \pi i}{3}\left(B_{3}(-A)+B_{3}(A)\right) \tau^{2}+2 \pi i A z \tau-\pi i A \tau \\
& =-\pi i A(2(A \tau-z)+1) \tau=-\pi i A(2 \hat{A}+1) \tau
\end{aligned}
$$

and

$$
\begin{aligned}
\tau F_{-}(\hat{z} ; \hat{\tau})-F_{-}(z ; \tau) & =\tau(\ln \overline{Q(-z ; \tau)}+\ln \overline{Q(z ; \tau)}) \\
& =-\frac{2 \pi i}{3}\left(B_{3}(-A)+B_{3}(A)\right) \tau \bar{\tau}-2 \pi i A \bar{z} \tau+\pi i A \tau \\
& =\pi i A(2(A \bar{\tau}-\bar{z})+1) \tau=\pi i A(2 \hat{A}+1) \tau
\end{aligned}
$$

We summarise our finding in the following claim.

Lemma 4 We have

$$
\begin{aligned}
& \tau F_{+}(\hat{z} ; \hat{\tau})-F_{+}(z ; \tau)=-\pi i A(2 \hat{A}+1) \tau, \\
& \tau F_{-}(\hat{z} ; \hat{\tau})-F_{-}(z ; \tau)=\pi i A(2 \hat{A}+1) \tau .
\end{aligned}
$$

Lemma 3 leads to the following expansions of the functions $F_{+}$and $F_{-}$. 
Theorem 1 We have

$$
\begin{aligned}
& F_{+}(z ; \tau)=S(z, \tau)-\frac{1}{2 \pi i} L(z, \tau), \\
& F_{-}(z ; \tau)=-\frac{2 \pi i \bar{\tau}(\tau-\bar{\tau})}{3} B_{3}(A)+\overline{S(z, \tau)}+\frac{1}{\pi} \overline{U(z, \tau)}+\frac{1}{2 \pi i} \overline{L(z, \tau)},
\end{aligned}
$$

where

$$
\begin{aligned}
& L(z, \tau):=\sum_{m=0}^{\infty}\left(\operatorname{Li}_{2}\left(x^{-1} q^{m+1}\right)-\operatorname{Li}_{2}\left(x q^{m}\right)\right) \\
& U(z, \tau):=\sum_{m=0}^{\infty}\left(\ln \left|x^{-1} q^{m+1}\right| \operatorname{Li}_{1}\left(x^{-1} q^{m+1}\right)-\ln \left|x q^{m}\right| \operatorname{Li}_{1}\left(x q^{m}\right)\right) \\
& S(z, \tau):=\frac{-\pi i}{12}(2 A-1)\left(6 z^{2}-12 A \tau z+6 z+8 A^{2} \tau^{2}-2 A \tau^{2}-6 A \tau+1\right)
\end{aligned}
$$

Proof For $F_{+}$substitute the expression of $T(z ; \tau)$ from Lemma 3 into the computation

$$
\begin{aligned}
F_{+}(z ; \tau)= & \ln Q(\hat{z} ; \hat{\tau})-\tau \ln Q(z ; \tau) \\
= & \frac{2 \pi i}{3}\left(B_{3}(\hat{A}) \hat{\tau}-B_{3}(A) \tau^{2}\right)+\hat{A} \ln \theta_{0}(\hat{z} ; \hat{\tau})-(\hat{A}+z) \ln \theta_{0}(z ; \tau) \\
& +\tau \ln \theta_{1}(z ; \tau)-\ln \theta_{1}(\hat{z} ; \hat{\tau}) .
\end{aligned}
$$

This leads to the formula

$$
F_{+}(z ; \tau)=S(z, \tau)-\frac{1}{2 \pi i} L(z, \tau)
$$

with

$$
\begin{aligned}
S(z, \tau)= & \frac{2 \pi i}{3}\left(B_{3}(\hat{A}) \hat{\tau}-B_{3}(A) \tau^{2}\right)+\hat{A} \pi i\left(\frac{\tau}{6}-\frac{\hat{\tau}}{6}+z \hat{z}-\frac{1}{2}-z+\hat{z}\right) \\
& +\frac{\pi i}{12 \tau}(\tau-2 z)\left(1+2 \tau z-2 z^{2}\right),
\end{aligned}
$$

and the latter simplifies to the expression given in the statement of Theorem 1 by elementary manipulation.

For $F_{-}$we proceed as follows. We have

$$
\ln Q(z ; \tau)=\frac{2 \pi i \tau B_{3}(A)}{3}-\sum_{m=0}^{\infty}\left((m+1-A) \operatorname{Li}_{1}\left(x^{-1} q^{m+1}\right)-(m+A) \operatorname{Li}_{1}\left(x q^{m}\right)\right) .
$$

Multiply this expression by $\tau-\bar{\tau}=2 i \operatorname{Im} \tau$ and use $A(\tau-\bar{\tau})=2 i \operatorname{Im} z$ to get

$$
(\tau-\bar{\tau}) \ln Q(z ; \tau)=\frac{2 \pi i \tau(\tau-\bar{\tau}) B_{3}(A)}{3}-\frac{1}{\pi} U(z, \tau) .
$$

Now, notice

$$
\overline{(\tau-\bar{\tau}) \ln Q(z ; \tau)}=F_{-}(z ; \tau)-\overline{F_{+}(z ; \tau)}
$$

to deduce the expression for $F_{-}$as in the theorem.

A consequence of this expansion is the invariance of

$$
F(z ; \tau):=\frac{F_{+}(z ; \tau)+F_{-}(z ; \tau)}{2}=\ln |Q(\hat{z} ; \hat{\tau})|-\tau \ln |Q(z ; \tau)|
$$

under translation $\tau \mapsto \tau+1$. 
Lemma 5 We have

$$
F_{+}(z ; \tau+1)-F_{+}(z ; \tau)=-\left(F_{-}(z ; \tau+1)-F_{-}(z ; \tau)\right) .
$$

Proof The functions $L(z, \tau)$ and $U(z, \tau)$ (hence their complex conjugates) are clearly invariant under translation $\tau \mapsto \tau+1$. The result follows from noticing that

$$
\begin{aligned}
2 \operatorname{Re} S(z, \tau)+\frac{2 \pi i \bar{\tau}(\tau-\bar{\tau})}{3} B_{3}(A) & =\frac{-\pi i(\tau-\bar{\tau})^{2} A(1-A)(1-2 A)}{6} \\
& =\frac{-\pi i(\tau-\bar{\tau})^{2}}{3} B_{3}(A)
\end{aligned}
$$

is also invariant under the transformation.

We summarise the results in this section as follows.

Theorem 2 The weight 1 period function

$$
\begin{aligned}
F(z ; \tau)= & \ln |Q(\hat{z} ; \hat{\tau})|-\tau \ln |Q(z ; \tau)| \\
= & \frac{1}{2 \pi} \sum_{m=0}^{\infty}\left(\ln \left|x^{-1} q^{m+1}\right| \overline{\operatorname{Li}_{1}\left(x^{-1} q^{m+1}\right)}-\ln \left|x q^{m}\right| \overline{\operatorname{Li}_{1}\left(x q^{m}\right)}\right) \\
& -\frac{\pi i(\tau-\bar{\tau})^{2}}{6} B_{3}(A)-\frac{1}{2 \pi i} \operatorname{Im} \sum_{m=0}^{\infty}\left(\operatorname{Li}_{2}\left(x^{-1} q^{m+1}\right)-\operatorname{Li}_{2}\left(x q^{m}\right)\right)
\end{aligned}
$$

of $\ln |Q(z ; \tau)|$ satisfies

$$
\tau F(\hat{z} ; \hat{\tau})=F(z ; \tau) \text { and } F(z ; \tau)=F(z ; \tau+1) .
$$

In other words, it behaves like a Jacobi form of weight 1 on $\mathrm{SL}_{2}(\mathbb{Z})$.

\section{Elliptic dilogarithm}

Theorem 2 provides a natural link between the period function $F(z ; \tau)$ and the elliptic dilogarithm [7]

$$
D(q ; x):=\sum_{m \in \mathbb{Z}} D\left(x q^{m}\right)=\sum_{m=0}^{\infty}\left(D\left(x q^{m}\right)-D\left(x^{-1} q^{m+1}\right)\right)
$$

together with its companion

$$
J(q ; x):=\sum_{m=0}^{\infty}\left(J\left(x q^{m}\right)-J\left(x^{-1} q^{m+1}\right)\right)+\frac{\log ^{2}|q|}{3} B_{3}\left(\frac{\log |x|}{\log |q|}\right),
$$

where

$$
D(x):=\ln |x| \arg (1-x)+\operatorname{Im} \operatorname{Li}_{2}(x)=-\ln |x| \operatorname{Im~Li}_{1}(x)+\operatorname{Im} \operatorname{Li}_{2}(x)
$$

denotes the Bloch-Wigner dilogarithm and

$$
J(x):=\ln |x| \ln |1-x|=-\ln |x| \operatorname{ReLi}_{1}(x)
$$

its companion. Namely, the expansion in the theorem can be stated as

$$
F(z ; \tau)=\frac{1}{2 \pi i}(D(q ; x)+i J(q ; x)) .
$$


This is essentially the result discussed in [2, Section 1].

Viewing now $z$ as an element of the lattice $\mathbb{R}+\mathbb{R} \tau$, so that $A$ and $\hat{A}$ in the representation $z=-\hat{A}+A \tau$ are fixed, we find out that the $\tau$-derivative

$$
\frac{1}{2 \pi i} \frac{\mathrm{d}}{\mathrm{d} \tau} \ln Q(z ; \tau)=q \frac{\mathrm{d}}{\mathrm{d} q} \ln Q(z ; \tau)
$$

is the Eisenstein series

$$
\frac{i}{4 \pi^{3}} \sum_{m, n \in \mathbb{Z}}^{\prime} \frac{e^{2 \pi i(m \hat{A}+n A)}}{(m \tau+n)^{3}}
$$

of weight 3 , where the notation $\sum^{\prime}$ indicates omitting the term $m=n=0$ from the summation. Integrating we obtain

$$
\ln Q(z ; \tau)=\frac{1}{4 \pi^{2}} \sum_{m, n \in \mathbb{Z}}^{\prime} \frac{e^{2 \pi i(m \hat{A}+n A)}}{m(m \tau+n)^{2}}
$$

implying

$$
\begin{aligned}
\ln |Q(z ; \tau)| & =\frac{1}{2}(\ln Q(z ; \tau)+\ln \overline{Q(z ; \tau)}) \\
& =\frac{1}{8 \pi^{2}} \sum_{m, n \in \mathbb{Z}}^{\prime} e^{2 \pi i(m \hat{A}+n A)}\left(\frac{1}{m(m \tau+n)^{2}}-\frac{1}{m(m \bar{\tau}+n)^{2}}\right) \\
& =\frac{1}{2 \pi^{2}} \sum_{m, n \in \mathbb{Z}}^{\prime} e^{2 \pi i(m \hat{A}+n A)} \frac{i m \operatorname{Im} \tau(m \operatorname{Re} \tau+n)}{m(m \tau+n)^{2}(m \bar{\tau}+n)^{2}} \\
& =\frac{i \operatorname{Im} \tau}{2 \pi^{2}} \sum_{m, n \in \mathbb{Z}}^{\prime} \frac{e^{2 \pi i(m \hat{A}+n A)}(m \operatorname{Re} \tau+n)}{|m \tau+n|^{4}}
\end{aligned}
$$

This is equation (7) in [2]. Since $\hat{z}=z / \tau=A-\hat{A} / \tau=A+\hat{A} \hat{\tau}$, it follows that

$$
\begin{aligned}
\ln |Q(\hat{z} ; \hat{\tau})| & =\frac{i \operatorname{Im} \hat{\tau}}{2 \pi^{2}} \sum_{m, n \in \mathbb{Z}}^{\prime} \frac{e^{2 \pi i(-m A+n \hat{A})}(m \operatorname{Re} \hat{\tau}+n)}{|m \hat{\tau}+n|^{4}} \\
& =\frac{i \operatorname{Im} \tau}{2 \pi^{2}|\tau|^{2}} \sum_{m, n \in \mathbb{Z}}^{\prime} \frac{e^{2 \pi i(n \hat{A}-m A)}\left(-m(\operatorname{Re} \tau) /|\tau|^{2}+n\right)}{|n-m / \tau|^{4}} \\
& =\frac{i \operatorname{Im} \tau}{2 \pi^{2}} \sum_{m, n \in \mathbb{Z}}^{\prime} \frac{e^{2 \pi i(n \hat{A}-m A)}\left(n|\tau|^{2}-m \operatorname{Re} \tau\right)}{|n \tau-m|^{4}} \\
& =\frac{i \operatorname{Im} \tau}{2 \pi^{2}} \sum_{m, n \in \mathbb{Z}}^{\prime} \frac{e^{2 \pi i(m \hat{A}+n A)}\left(m|\tau|^{2}+n \operatorname{Re} \tau\right)}{|m \tau+n|^{4}} \\
& =\frac{\operatorname{Im} \tau}{2 \pi^{2}} \sum_{m, n \in \mathbb{Z}}^{\prime} \frac{e^{2 \pi i(m \hat{A}+n A)}((m \operatorname{Re} \tau+n) \tau i+(m \tau+n) \operatorname{Im} \tau)}{|m \tau+n|^{4}}
\end{aligned}
$$

implying

$$
\ln |Q(\hat{z} ; \hat{\tau})|-\tau \ln |Q(z ; \tau)|=\frac{(\operatorname{Im} \tau)^{2}}{2 \pi^{2}} \sum_{m, n \in \mathbb{Z}}^{\prime} \frac{e^{2 \pi i(m \hat{A}+n A)}(m \tau+n)}{|m \tau+n|^{4}} .
$$

The latter is a (non-holomorphic) modular form of weight 1 , and combined with equation (6) is the formula of Bloch mentioned previously. 
Theorem 3 (Bloch's formula $[1,2,7]$ ) For $z=A \tau-\hat{A}$, we have

$$
\begin{aligned}
F(z ; \tau) & =\frac{1}{2 \pi i}(D(q ; x)+i J(q ; x)) \\
& =\frac{(\operatorname{Im} \tau)^{2}}{2 \pi^{2}} \sum_{m, n \in \mathbb{Z}} \frac{e^{2 \pi i(m \hat{A}+n A)}(m \tau+n)}{|m \tau+n|^{4}} .
\end{aligned}
$$

\section{General weight}

A natural generalisation of the product in (5) is

$$
Q_{k}(z ; \tau):=q^{B_{k+2}(A) /(k+2)} \prod_{m=0}^{\infty}\left(1-x q^{m}\right)^{(m+A)^{k}}\left(1-x^{-1} q^{m+1}\right)^{(-1)^{k}(m+1-A)^{k}},
$$

where $k=0,1,2, \ldots$ and $B_{k}(t)$ stands for the $k$ th Bernoulli polynomial. Then $Q_{0}(z ; \tau)$ is an arithmetic normalisation of the short theta function $\theta_{0}(z ; \tau)$ (a Siegel modular unit) and $Q_{1}(z ; \tau)$ coincides with (5). Following the earlier recipe, define

$$
\begin{aligned}
& F_{+}(z ; \tau)=F_{k,+}(z ; \tau):=\ln Q_{k}(\hat{z} ; \hat{\tau})-\tau^{k-2} \ln Q_{k}(z ; \tau), \\
& F_{-}(z ; \tau)=F_{k,-}(z ; \tau):=\ln \overline{Q_{k}(\hat{z} ; \hat{\tau})}-\tau^{k-2} \ln \overline{Q_{k}(z ; \tau)}
\end{aligned}
$$

and $F_{k}(z ; \tau):=\frac{1}{2}\left(F_{k,+}(z ; \tau)+F_{k,-}(z ; \tau)\right)$. Then from Lemma 1 we deduce the following generalisation of Lemma 4 .

Lemma 6 We have, for $k \geq 1$,

$$
\begin{aligned}
& \tau^{k} F_{+}(\hat{z} ; \hat{\tau})+(-1)^{k} F_{+}(z ; \tau)=(-1)^{k} \pi i A^{k}(2 \hat{A}+1) \tau^{k}, \\
& \tau^{k} F_{-}(\hat{z} ; \hat{\tau})+(-1)^{k} F_{-}(z ; \tau)=-(-1)^{k} \pi i A^{k}(2 \hat{A}+1) \tau^{k} .
\end{aligned}
$$

Proof Apply Lemma 1 and the relation

$$
B_{k+2}(-t)-(-1)^{k} B_{k+2}(t)=(-1)^{k}(k+2) t^{k+1} .
$$

We further use that the $\tau$-derivative of $\ln Q_{k}(z ; \tau)$ is an Eisenstein series.

Lemma 7 For $k \geq 1$,

$$
\ln Q_{k}(z ; \tau)=\frac{(-1)^{k} k !}{(2 \pi i)^{k+1}} \sum_{m, n \in \mathbb{Z}}^{\prime} \frac{e^{2 \pi i(m \hat{A}+n A)}}{m(m \tau+n)^{k+1}},
$$

where $z=-\hat{A}+A \tau$.

Proof Consider $\tilde{Q}_{k}(A, \hat{A} ; \tau):=Q_{k}(A \tau-\hat{A} ; \tau)$ as a function of real variables $A, \hat{A}$ and complex variable $\tau$. The $\tau$-derivative

$$
G_{k+2}(A, \hat{A} ; \tau):=\frac{1}{2 \pi i} \frac{\mathrm{d}}{\mathrm{d} \tau} \ln Q_{k}(A, \hat{A} ; \tau)=q \frac{\mathrm{d}}{\mathrm{d} q} \ln Q_{k}(A, \hat{A} ; \tau)
$$

is seen to be the Eisenstein series

$$
E_{k+2}(A, \hat{A} ; \tau):=\frac{(-1)^{k+1}(k+1) !}{(2 \pi i)^{k+2}} \sum_{m, n \in \mathbb{Z}}^{\prime} \frac{e^{2 \pi i(m \hat{A}+n A)}}{(m \tau+n)^{k+2}}
$$


of weight $k+2$. This is true for $k=1$ (see Sect. 4), while for $k \geq 1$ we observe the functional equation

$$
\frac{\partial}{\partial \hat{A}} E_{k+3}(A, \hat{A} ; \tau)=\frac{\partial}{\partial \tau} E_{k+2}(A, \hat{A} ; \tau) .
$$

The equality $G_{k+2}(A, \hat{A} ; \tau)=E_{k+2}(A, \hat{A} ; \tau)$ then follows by induction on $k$ using the fact that the constant terms of both functions at $\tau=\infty$ (or $q=0$ ) agree.

Integrating we obtain

$$
\ln Q_{k}(A, \hat{A} ; \tau)=\frac{(-1)^{k} k !}{(2 \pi i)^{k+1}} \sum_{m, n \in \mathbb{Z}}^{\prime} \frac{e^{2 \pi i(m \hat{A}+n A)}}{m(m \tau+n)^{k+1}} .
$$

Since both sides continuously depend on $A$ and $\hat{A}$, the formula remains valid also for $\ln Q_{k}(z ; \tau)$.

As in our computation in Sect. 4 we obtain

$$
\begin{aligned}
\ln \left|Q_{k}(z ; \tau)\right| & =\frac{(-1)^{k} k !}{2(2 \pi i)^{k+1}} \sum_{m, n \in \mathbb{Z}}^{\prime} e^{2 \pi i(m \hat{A}+n A)}\left(\frac{1}{m(m \tau+n)^{k+1}}-\frac{1}{m(m \bar{\tau}+n)^{k+1}}\right) \\
& =\frac{(-1)^{k} k !}{2(2 \pi i)^{k+1}} \sum_{m, n \in \mathbb{Z}}^{\prime} \frac{e^{2 \pi i(m \hat{A}+n A)}(\bar{\tau}-\tau)}{(m \tau+n)^{k+1}(m \bar{\tau}+n)^{k+1}} \sum_{j=0}^{k}(m \tau+n)^{j}(m \bar{\tau}+n)^{k-j} \\
& =-\frac{i^{k} k ! \operatorname{Im} \tau}{(2 \pi)^{k+1}} \sum_{j=0}^{k} \sum_{m, n \in \mathbb{Z}}^{\prime} \frac{e^{2 \pi i(m \hat{A}+n A)}}{(m \tau+n)^{k-j+1}(m \bar{\tau}+n)^{j+1}}
\end{aligned}
$$

and

$$
\begin{aligned}
\ln \left|Q_{k}(\hat{z} ; \hat{\tau})\right| & =-\frac{i^{k} k ! \operatorname{Im} \tau}{(2 \pi)^{k+1}|\tau|^{2}} \sum_{j=0}^{k} \sum_{m, n \in \mathbb{Z}}^{\prime} \frac{e^{2 \pi i(-m A+n \hat{A})}}{(n-m / \tau)^{j+1}(n-m / \bar{\tau})^{k-j+1}} \\
& =-\frac{i^{k} k ! \operatorname{Im} \tau}{(2 \pi)^{k+1}} \sum_{j=0}^{k} \sum_{m, n \in \mathbb{Z}}^{\prime} \frac{e^{2 \pi i(m \hat{A}+n A)} \tau^{k-j} \bar{\tau}^{j}}{(m \tau+n)^{k-j+1}(m \bar{\tau}+n)^{j+1}} .
\end{aligned}
$$

Thus,

$$
\begin{aligned}
F_{k}(z ; \tau) & =\ln \left|Q_{k}(\hat{z} ; \hat{\tau})\right|-\tau^{k} \ln \left|Q_{k}(z ; \tau)\right| \\
& =\frac{i^{k} k ! \operatorname{Im} \tau}{(2 \pi)^{k+1}} \sum_{j=0}^{k} \tau^{k-j}\left(\tau^{j}-\bar{\tau}^{j}\right) \sum_{m, n \in \mathbb{Z}}^{\prime} \frac{e^{2 \pi i(m \hat{A}+n A)}}{(m \tau+n)^{j+1}(m \bar{\tau}+n)^{k-j+1}} \\
& =\frac{i^{k} k !}{2(2 \pi)^{k}(\tau-\bar{\tau})^{k}} \sum_{j=1}^{k} \tau^{k-j}\left(\tau^{j}-\bar{\tau}^{j}\right) D_{j+1, k-j+1}(q ; x) \\
& =\frac{i k !}{(4 \pi \operatorname{Im} \tau)^{k}} \sum_{j=1}^{k} \tau^{k-j} \operatorname{Im}\left(\tau^{j}\right) D_{j+1, k-j+1}(q ; x),
\end{aligned}
$$

where

$$
D_{a, b}(q ; x):=\frac{(\tau-\bar{\tau})^{a+b-1}}{2 \pi i} \sum_{m, n \in \mathbb{Z}}^{\prime} \frac{e^{2 \pi i(m \hat{A}+n A)}}{(m \tau+n)^{a}(m \bar{\tau}+n)^{b}}
$$


for positive integers $a$ and $b$.

Finally, observe that the non-holomorphic Eisenstein series (8) can be identified with the elliptic polylogarithms using a formula of Zagier [7, Proposition 2]. This leads to the following general result.

Theorem 4 For $k \geq 1$ and $z=A \tau-\hat{A}$, we have

$$
\ln \left|Q_{k}(\hat{z} ; \hat{\tau})\right|-\tau^{k} \ln \left|Q_{k}(z ; \tau)\right|=\frac{i k !}{(4 \pi \operatorname{Im} \tau)^{k}} \sum_{j=1}^{k} \tau^{k-j} \operatorname{Im}\left(\tau^{j}\right) D_{j+1, k-j+1}(q ; x),
$$

where

$$
D_{a, b}(q ; x)=\sum_{m=0}^{\infty}\left(D_{a, b}\left(x q^{m}\right)+(-1)^{a+b} D_{a, b}\left(x^{-1} q^{m+1}\right)\right)+\frac{(4 \pi \operatorname{Im} \tau)^{a+b-1}}{(a+b) !} B_{a+b}(A)
$$

and

$$
\begin{aligned}
D_{a, b}(x)= & (-1)^{a-1} \sum_{\ell=a}^{a+b-1} 2^{a+b-\ell-1}\left(\begin{array}{l}
\ell-1 \\
a-1
\end{array}\right) \frac{(-\ln |x|)^{a+b-\ell-1}}{(a+b-\ell-1) !} \operatorname{Li}_{\ell}(x) \\
& +(-1)^{b-1} \sum_{\ell=b}^{a+b-1} 2^{a+b-\ell-1}\left(\begin{array}{l}
\ell-1 \\
b-1
\end{array}\right) \frac{(-\ln |x|)^{a+b-\ell-1}}{(a+b-\ell-1) !} \overline{\operatorname{Li}_{\ell}(x)}
\end{aligned}
$$

\section{Conclusion}

This final (and very short!) part is devoted to highlighting some directions for further research.

In spite of generalisability of the story in Sects. $2-4$ to the function

$$
F_{k}(z ; \tau)=\ln \left|Q_{k}(\hat{z} ; \hat{\tau})\right|-\tau^{k} \ln \left|Q_{k}(z ; \tau)\right|,
$$

where $k \geq 1$ and the product $Q_{k}(z ; \tau)$ is defined in (7), the case $k=1$ remains the only one, which is invariant under translation $\tau \mapsto \tau+1$. At the same time, Lemma 6 implies the transformation

$$
\tau^{k} F_{k}(\hat{z}, \hat{\tau})=(-1)^{k-1} F_{k}(z, \tau) \text { for } k=1,2, \ldots
$$

This consideration does not exclude, however, a possibility for modified products (7) and related functions $F_{k}$ to exist such that the latter ones have true modular behaviour for each $k \geq 1$. It sounds to us a nice problem to determine such modular objects.

Several arithmetic problems related to the case $k=1$ (originating from the elliptic gamma function) are still open. Our personal favourites include connection of (5) with the Mahler measure and mirror symmetry; see, for example, observation in [6].

\footnotetext{
Author details

${ }^{1}$ Simion Stoilow Institute of Mathematics of the Romanian Academy, P.O. Box 1-764, 014700 Bucharest, Romania, ${ }^{2}$ Department of Mathematics, IMAPP, Radboud University, P.O. Box 9010, 6500 GL Nijmegen, The Netherlands, ${ }^{3}$ School of Mathematical and Physical Sciences, The University of Newcastle, Callaghan, NSW 2308, Australia, ${ }^{4}$ Laboratory of Mirror Symmetry and Automorphic Forms, National Research University Higher School of Economics, 6 Usacheva str., 119048 Moscow, Russia.
}

\section{Acknowledgements}

We thank the anonymous referees for their careful reading of the manuscript and reporting valuable feedback. The first author was partially supported by a grant of Romanian Ministry of Research and Innovation, CNCS - UEFISCDI, Project Number PN-III-P4-ID-PCE-2016-0157, within PNCDI III7. The second author is partially supported by Laboratory of Mirror Symmetry NRU HSE, RF Government Grant, Ag. No. 14.641.31.0001. 


\section{Conflict of interest}

On behalf of all authors, the corresponding author Wadim Zudilin states that there is no conflict of interest.

Received: 30 December 2017 Accepted: 4 May 2018 Published online: 28 September 2018

\section{References}

1. Bloch, S.J.: Higher regulators, algebraic K-theory, and zeta functions of elliptic curves. In: Lecture Notes (UC Irvine, 1977); CRM Monograph Series, vol. 11. American Mathematical Society, Providence (2000)

2. Duke, W., Imamoğlu, Ö.: On a formula of Bloch. Funct. Approx. 37(1), 109-117 (2007)

3. Felder, G., Varchenko, A.: The elliptic gamma function and $S L(3, \mathbb{Z}) \ltimes \mathbb{Z}^{3}$. Adv. Math. 156(1), 44-76 (2000)

4. Felder, G., Varchenko, A.: Multiplication formulae for the elliptic gamma function. In: Fuchs, J., Mickelsson, J., Rozenblioum, G., Stolin, A., Westerberg, A. (eds.) Noncommutative Geometry and Representation Theory in Mathematical Physics, Contemporary Mathematics, vol. 391, pp. 69-73. American Mathematical Society, Providence (2005)

5. Ruijsenaars, S.: First order analytic difference equations and integrable quantum systems. J. Math. Phys. 38, 1069-1146 (1997)

6. Stienstra, J.: Mahler measure variations, Eisenstein series and instanton expansions. In: Yui, N., Yau, S.-T., Lewis, J.D. (eds.) Mirror Symmetry V. AMS/IP Studies in Advanced Mathematics, vol. 38, pp. 139-150. American Mathematical Society, Providence (2006)

7. Zagier, D.: The Bloch-Wigner-Ramakrishnan polylogarithm function. Math. Ann. 286, 613-624 (1990) 